\title{
Comparison of the Statistical Properties of the LTE-A and IMT-A Channel Models
}

\author{
Qi Yao ${ }^{1}$, Yi Yuan ${ }^{1}$, Ammar Ghazal ${ }^{1}$, Cheng-Xiang Wang ${ }^{1}$, Longyuan $\mathrm{Luan}^{2}$, and Xiaofeng $\mathrm{Lu}^{2}$ \\ ${ }^{1}$ Joint Research Institute for Signal and Image Processing, Heriot-Watt University, Edinburgh EH14 4AS, UK. \\ ${ }^{2}$ Huawei Technologies Co., Ltd., Xi' an, 710075, China. \\ Email: \{q.yao, yy120, ag289, cheng-xiang.wang\}@hw.ac.uk, \{luanlongyuan, stan.x.lu\}@ huawei.com
}

\begin{abstract}
For the design and performance evaluation of advanced wireless communication systems employing multipleinput multiple-output (MIMO) technologies, realistic MIMO channel models with a good tradeoff between accuracy and complexity are indispensable. This paper compares the statistical properties of the two latest standardized MIMO channel models: Long Term Evolution-Advanced (LTE-A) and IMT-Advanced (IMT-A) channel models. Closed-from expressions are derived for the spatial cross-correlation function (CCF), temporal autocorrelation function (ACF), envelope level-crossing rate (LCR), average fading duration (AFD), power delay profile (PDP), and frequency correlation function (FCF) for both models. Simulation results are provided which can match the corresponding theoretical derivations very well, demonstrating the correctness of both theoretical and simulation results. The LTE-A channel model is simple but has significant flaws in terms of the accuracy. It can only support system bandwidths up to $50 \mathrm{MHz}$, not the claimed $100 \mathrm{MHz}$, and only describes the average spatialtemporal properties of MIMO channels. The IMT-A channel model is complex with more model parameters but has better accuracy. It allow us to simulate the variations of different MIMO channel realizations and can indeed support system bandwidths up to $100 \mathrm{MHz}$.
\end{abstract}

\section{INTRODUCTION}

The employment of multiple antennas at both the transmitter (Tx) and receiver ( $\mathrm{Rx})$ enables the so-called MIMO technologies to greatly improve the link reliability and increase the overall system capacity [1], [2]. MIMO has been widely used or recommended to be used in various standards, such as the third generation $(3 \mathrm{G})$ and the fourth generation $(4 \mathrm{G})$ wireless communication systems. To evaluate the performance of candidate technologies for 3GPP LTE, LTE-A, and IMTA communication systems, realistic MIMO channel models are indispensable. This requires a good tradeoff between the model accuracy and complexity, i.e., it must accurately reflect important statistical properties of real MIMO propagation channels with reasonable computational complexity. Among other features, accurate MIMO channel models should at least consider spatial-temporal correlation properties and channel variations of multiple users/links and multiple cells at the system level, instead of only at the link level. Inaccurate or over-simplified channel models may lead to either too optimistic or too pessimistic performance evaluation results of chosen transmission schemes.

The standardized MIMO channel models can roughly be classified into geometry-based stochastic models (GB$\mathrm{SMs}$ ) and correlation-based stochastic models (CBSMs) or
Kronecker-based stochastic models (KBSMs). A KBSM assumes that the channel coefficients are complex Gaussian distributed and therefore, the first-order and second-order statistical properties can fully characterize the channel behavior [3]. It also assumes the separability of the correlations between the $\mathrm{Tx}$ and $\mathrm{Rx}$ so that the spatial correlation matrix of the channel can be expressed as the Kronecker product of the Tx and Rx correlation matrices. This assumption also implies the independence between the angle-of-arrivals (AoAs) and angle-of-departures (AoDs), which is unrealistic in certain scenarios like in pico- and micro-cells. The IEEE 802.11 TGn channel model [4], LTE channel model [5]-[7], and LTE-A channel model [8], all belong to KBSMs. A GBSM characterizes the propagation environment using a geometric description. Standardized GBSMs are often characterized by using selected random parameters such as AoA, AoD, and propagation delay. They all adopt the sum-of-sinusoids (SoS) based double directional channel modeling approach. The 3GPP/3GPP2 spatial channel model (SCM) [9], WINNER channel Model (WIN)-Phase II (WIM2) [10], and IMT-A channel model [11], [12], only name a few, belong to GBSMs.

In [13], the spatial-temporal correlation properties of the SCM [9] and a typical KBSM [3] were compared in a great detail. However, in [13] other important statistical properties, e.g., envelope LCRs, AFDs, PDPs, and FCFs of the GBSMs and KBSMs were not analyzed and compared. The LTE-A and IMT-A channel models represent the latest developments of the standardized KBSM and GBSM, respectively. To the best of our knowledge, no one has studied in detail and compared the statistical properties of both models. The aim of this paper is to fill this research gap.

The rest of this paper is organized as follows. The LTE-A and IMT-A MIMO channel models are briefly described in Sections II and III, respectively. In Section IV the statistical properties of the LTE-A and IMT-A channel models are fully investigated and compared. The conclusions are drawn in Section V.

\section{The LTE-A MIMO CHANNEL MODEL}

The LTE-A channel model [8] makes the following assumptions that are not yet well-justified. The same spatial correlation matrix is applied to all the resolvable paths/taps, which indicates that all the taps have the same spatial correlation properties. The spatial correlation matrix of the MIMO channel 
is given by the Kronecker product of the Tx correlation matrix and $\mathrm{Rx}$ correlation matrix, implying the assumption of independence between the AoA and AoD. The spatial correlation matrix is assumed to be time-invariant and independent of the Doppler power spectrum density.

\section{THE IMT-A CHANNEL MODEL}

Based on the WIM2, the IMT-A [12] channel model still uses two types of channel models, namely a generic model and clustered delay line (CDL) model. The generic model is a double directional GBSM that describes the geometric distribution of the scatterers considering the arrival angles from the last bounce scatterers and the departure angles to the first scatterers involved from the Tx side, and enables the separation of propagation parameters and antennas. This model is mainly for system level simulation purposes, while the CDL model is a spatial extension of the tapped delay line (TDL) model for calibration use only. The reduced variability of CDL has been achieved through fixing all of the parameters except for the phases of the rays.

\section{Statistical Properties of the LTE-A AND IMT-A Channel Models}

In this paper, the base station (BS) and mobile station (MS) are used which refer to the eNode B (eNB) and user equipment (UE) in [8], respectively. Considering a downlink transmission system with an $S$ element linear BS array and a $U$ element linear MS array, in this section we will derive and compare some important statistical properties of the LTE-A MIMO channel model and IMT-A MIMO channel model based on the generic model, including spatial $\mathrm{CCF}$, temporal ACF, envelope LCR, AFD, PDP, and FCF.

\section{A. Statistical Properties of the LTE-A MIMO Channel Model}

1) Spatial-temporal CCF: The distance between BS and MS antenna elements are denoted as $\Delta d_{s}$ and $\Delta d_{u}$, respectively. The spatial CCF $\widehat{\rho}_{s_{2} u_{2}}^{s_{1} u_{1}}\left(\Delta d_{s}, \Delta d_{u}\right)$ between two arbitrary transmission coefficients is the product of the spatial CCF $\hat{\rho}_{s_{1} s_{2}}^{B S}\left(\Delta d_{s}\right)$ at the BS and the spatial CCF at the MS $\widehat{\rho}_{u_{2} u_{2}}^{M S_{2}}\left(\Delta d_{u}\right)$ [13], i.e., $\widehat{\rho}_{s_{2} u_{2}}^{s_{1} u_{1}}\left(\Delta d_{s}, \Delta d_{u}\right)=$ $\widehat{\rho}_{s_{1} s_{2}}^{B S}\left(\Delta d_{s}\right) \widehat{\rho}_{u_{2} u_{2}}^{M S_{2}}\left(\Delta d_{u}\right)$. The complex spatial CCF at the MS is given by [13]

$$
\widehat{\rho}_{u_{1} u_{2}}^{M S}\left(\Delta d_{u}\right)=\int_{0}^{2 \pi} e^{j k \Delta d_{u} \sin \left(\widehat{\varphi}_{A o A}\right)} p_{u}\left(\widehat{\varphi}_{A o A}\right) d \widehat{\varphi}_{A o A}
$$

where $\widehat{\varphi}_{A O A}$ is the AoA, $p_{u}\left(\widehat{\varphi}_{A o A}\right)$ denotes the power azimuth spectrum (PAS) of the absolute AoA, $\lambda$ denotes the carrier wavelength, and $k=2 \pi / \lambda$ is the wave number. The complex spatial CCF at the BS is given by [13]:

$$
\widehat{\rho}_{s_{1} s_{2}}^{B S}\left(\Delta d_{s}\right)=\int_{0}^{2 \pi} e^{j k \Delta d_{s} \sin \left(\widehat{\phi}_{A o D}\right)} p_{s}\left(\widehat{\phi}_{A o D}\right) d \widehat{\phi}_{A o D}
$$

where $\widehat{\phi}_{A o D}$ is the AoD and $p_{s}\left(\widehat{\phi}_{A o D}\right)$ denotes the PAS of the absolute AoD.

The temporal ACF is given by

$$
\widehat{r}\left(\tau^{\prime}\right)=J_{0}\left(2 \pi\|\mathbf{v}\| \tau^{\prime} / \lambda\right)
$$

where $J_{0}(x)$ is the Bessel function of the first kind of order zero, and $\tau^{\prime}$ and $\|\mathbf{v}\|$ denote the time difference and the magnitude of the MS velocity, respectively.

Because of the spatial temporal separability feature in the LTE-A channel model, the spatial-temporal CCF can be simply expressed as the product of the spatial $\mathrm{CCF}$ and the temporal $\mathrm{ACF}$, i.e.,

$$
\widehat{\rho}_{s_{2} u_{2}}^{s_{1} u_{1}}\left(\Delta d_{s}, \Delta d_{u}, \tau^{\prime}\right)=\widehat{\rho}_{s_{2} u_{2}}^{s_{1} u_{1}}\left(\Delta d_{s}, \Delta d_{u}\right) \widehat{r}\left(\tau^{\prime}\right) .
$$

2) Envelope LCR and AFD: The theoretical LCR for the LTE-A channel model follows the LCR for a Rayleigh model given by

$$
\widehat{N}_{h}(r)=\sqrt{2 \pi} f_{\max } r e^{-r^{2}}
$$

where $r=r_{E} / \sqrt{2 \sigma^{2}}$ is the normalized envelope level with $r_{E}$ denoting the envelope level and $f_{\max }=\|\mathbf{v}\| / \lambda$ is the maximum Doppler shift.

The AFD of the LTE-A channel model is given by

$$
\widehat{T}_{h}(r)=\frac{\lambda\left(e^{r^{2}}-1\right)}{\sqrt{2 \pi}\|\mathbf{v}\| r} .
$$

3) PDP and FCF: For TDL models, let us denote $L, \tau_{l}$ and $a_{l}^{2}$ as the number of taps, tap delay, and numerical power of the $l$ th path, respectively. The normalized PDP can be expressed as $\widehat{R}_{\tau}(\tau)=\frac{1}{X_{p}} \sum_{l=0}^{L-1} a_{l}^{2} \delta\left(\tau-\tau_{l}\right)$, where $X_{p}=\sum_{l=0}^{L-1} a_{l}^{2}$ is the total power of all taps. The normalized FCF $\widehat{\Psi}_{\tau}(\Delta f)$ is the Fourier transform of the normalized PDP $\widehat{\Psi}_{\tau}(\tau)$ and can be expressed as

$$
\widehat{\Psi}_{\tau}(\Delta f)=\frac{1}{X_{p}} \sum_{l=0}^{L-1} a_{l}^{2} \cdot e^{-j 2 \pi \Delta f \tau_{l}}
$$

where $\Delta f$ denotes the frequency spacing. In the following, we will highlight some important properties of the FCF $\widehat{\Psi}_{\tau}(\Delta f)$. From (7), we know that the $\operatorname{FCF} \widehat{\Psi}_{\tau}(\Delta f)$ is periodic with the period $\Upsilon$ given by $\Upsilon=\frac{1}{\operatorname{gcd}\left\{\tau_{0}, \tau_{1}, \tau_{L-1}\right\}}$ where $\operatorname{gcd}\{\cdot\}$ denotes the greatest common divisor. Therefore, we can write $\widehat{\Psi}_{\tau}(\Delta f)=\stackrel{\Psi}{\Psi}_{\tau}(\Delta f+\widehat{k} \cdot \Upsilon)$, where $\widehat{k}$ is an integer. Note that $\Upsilon \rightarrow \infty$ as $\operatorname{gcd}\left\{\tau_{0}, \tau_{1}, \tau_{L-1}\right\} \rightarrow 0$. Furthermore, it follows that the FCF exhibits the Hermitian symmetry property, i.e., $\widehat{\Psi}_{\tau}(\Delta f)=\widehat{\Psi}_{\tau}^{*}(-\Delta f)$. Till now, we can also conclude that $\widehat{\Psi}_{\tau}(\Delta f)=\widehat{\Psi}_{\tau}^{*}(\widehat{k} \cdot \Upsilon-\Delta f)$ and $\widehat{\Psi}_{\tau}([2 \widehat{k}+1] / 2 \cdot \Upsilon-\Delta f)=$ $\widehat{\Psi}_{\tau}^{*}([2 \widehat{m}+1] / 2 \cdot \Upsilon-\Delta f)$, where $\widehat{m}$ is also an integer. Thus, the real and imaginary parts of the FCF are even and odd functions, respectively, and the FCF is Hermitian symmetric with respect to a half of the period, i.e., to the value $\Delta f=$ $\Upsilon / 2$. Consequently, the complete information on the FCF is contained in a half of the period of the FCF. Finally, since $\widehat{\Psi}_{\tau}(\tau)=0$ for $\tau<0$, the real and imaginary parts of $\widehat{\Psi}_{\tau}(\Delta f)$ are related to each other by the Hilbert transform $\operatorname{Re}\left\{\widehat{\Psi}_{\tau}(\Delta f)\right\}=H\left\{\operatorname{Im}\left\{\widehat{\Psi}_{\tau}(\Delta f)\right\}\right\}$ where $\operatorname{Re}\{\cdot\}, \operatorname{Im}\{\cdot\}$, and $H\{\cdot\}$ denote the real part, imaginary part, and Hilbert transform, respectively. 


\section{B. Statistical Properties of the IMT-A MIMO Channel Model}

Fig. 1 illustrates the angular parameters in the model where the spatial angles are defined in a similar way to those as defined in the 3GPP SCM. However, in the IMT-A channel model the clusters with the total cluster number $N$ are further classified into two strongest clusters $(n=1,2)$ and $N-2$ weakest clusters $(n=3,4, \ldots, N)$. A strongest cluster still contains $M=20$ sub-paths but is subdivided into 3 subclusters, each of which contains $M_{q}(q=1,2,3)$ sub-paths. For the $n$th $(n=3,4, \ldots, N)$ weakest cluster, the relations $\varphi_{n, m}=\varphi_{n}+\Delta \varphi_{n, m}$ and $\phi_{n, m}=\phi_{n}+\Delta \phi_{n, m}$ hold for the AoA and AoD, respectively. Note that $\varphi_{n}, \Delta \varphi_{n, m}, \phi_{n}$, and $\Delta \phi_{n, m}$ denote the mean AoA, AoA offset, mean AoD, and AoD offset, respectively. For the $n$th $(n=1,2)$ strongest cluster and the $q$ th sub-cluster, the relations $\varphi_{n, q, m}=\varphi_{n, q}+$ $\Delta \varphi_{n, q, m}$ and $\phi_{n, q, m}=\phi_{n, q}+\Delta \phi_{n, q, m}$ hold for the AoA and AoD, respectively. Similarly, $\varphi_{n, q}, \Delta \varphi_{n, q, m}, \phi_{n, q}$, and $\Delta \phi_{n, q, m}$ denote the mean AoA, AoA offset, mean AoD, and AoD offset, respectively.

In case of the weakest clusters, without considering the antenna polarization the channel coefficients from the Tx antenna element $s$ to $\mathrm{Rx}$ antenna element $u$ for the cluster $n$ can be expressed as [12]

$$
\begin{aligned}
h_{u, s, n}(t)= & \sqrt{\frac{P_{n}}{M}} \sum_{m=1}^{M} e^{j d_{s} k \sin \left(\phi_{n, m}\right)} e^{j d_{u} k \sin \left(\varphi_{n, m}\right)} \\
& \cdot e^{j 2 \pi v_{n, m} t} e^{j \Phi_{n, m}} .
\end{aligned}
$$

Here, $h_{u, s, n}(t)$ denotes a narrowband process where all the $M$ sub-paths are irresolvable rays and have the same delay $\tau_{n}$. In (8), $P_{n}$ is the power of the $n$th cluster (path) associated with the delay $\tau_{n}$. The Doppler frequency component is $v_{n, m}=\lambda^{-1}\|\mathbf{v}\| \cos \left(\varphi_{n, m}-\theta_{v}\right)$, the random phases $\Phi_{n, m}$ are uniformly distributed within $[-\pi, \pi]$, and $\theta_{v}$ is the MS direction of travel.

1) Spatial-temporal CCF: The normalized spatial-temporal CCF $\rho_{s_{2} u_{2}}^{s_{1} u_{1}}\left(\Delta d_{s}, \Delta d_{u}, \tau^{\prime}\right)$ between two arbitrary channel coefficients connecting two different sets of antenna elements $\left(s_{1}-u_{1}\right.$ and $\left.s_{2}-u_{2}\right)$ is defined as

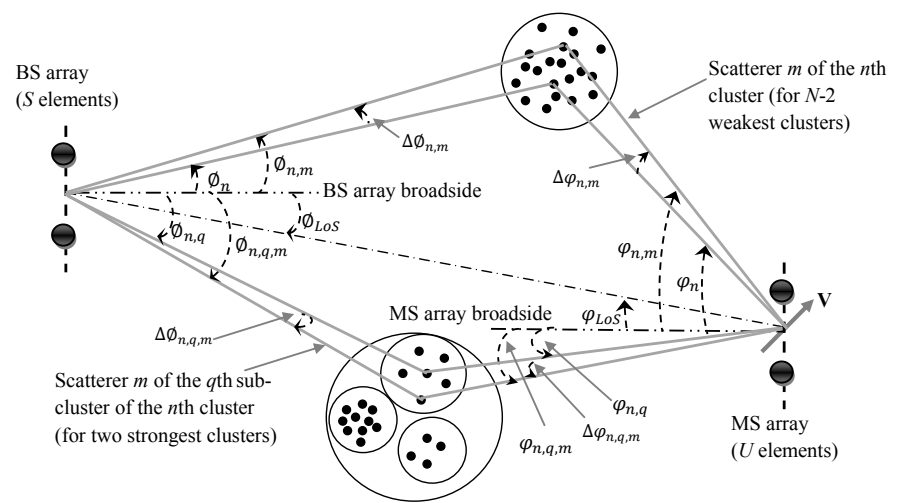

Fig. 1. The BS and MS angular parameters in the IMT-A channel model.

$$
\rho_{s_{2} u_{2}}^{s_{1} u_{1}}\left(\Delta d_{s}, \Delta d_{u}, \tau^{\prime}\right)=E\left\{\frac{h_{u_{1}, s_{1}, n}(t) h_{u_{2}, s_{2}, n}^{*}\left(t+\tau^{\prime}\right)}{\sigma_{h_{u_{1}, s_{1}, n}} \sigma_{h_{u_{2}, s_{2}, n}}}\right\}
$$

where $\Delta d_{s}=d_{s_{1}}-d_{s_{2}}$ is the distance between antenna elements $s_{1}$ and $s_{2}$ at the BS and $\Delta d_{u}=d_{u_{1}}-d_{u_{2}}$ is the distance between antenna elements $u_{1}$ and $u_{2}$ at the MS. Furthermore, $E(\cdot)$ is the assemble average operator, $h_{u_{2}, s_{2}, n}^{*}(t)$ is the complex conjugate of $h_{u_{2}, s_{2}, n}(t)$, and $\sigma_{h_{u_{i}, s_{i}, n}}=\sqrt{P_{n}}$ is the standard deviation of $h_{u_{i}, s_{i}, n}(t)$ for $i=1,2$. Substituting (8) into (9) results in

$$
\begin{aligned}
\rho_{s_{2} u_{2}}^{s_{1} u_{1}}\left(\Delta d_{s}, \Delta d_{u}, \tau^{\prime}\right)= & \frac{1}{M} \sum_{m=1}^{M} E\left\{e^{-j k\|\mathbf{v}\| \cos \left(\varphi_{n, m}-\theta_{v}\right) \tau^{\prime}}\right. \\
& \left.e^{j k\left[\Delta d_{s} \sin \left(\phi_{n, m}\right)+\Delta d_{u} \sin \left(\varphi_{n, m}\right]\right.}\right\} .(1
\end{aligned}
$$

By imposing $\tau^{\prime}=0$ in (10), we can get the spatial CCF $\rho_{s_{2} u_{2}}^{s_{1} u_{1}}\left(\Delta d_{s}, \Delta d_{u}\right)$ between two arbitrary channel coefficients at the same time instant:

$$
\begin{aligned}
& \rho_{s_{2} u_{2}}^{s_{1} u_{1}}\left(\Delta d_{s}, \Delta d_{u}\right) \\
= & \frac{1}{M} \sum_{m=1}^{M} E\left\{e^{j k\left[\Delta d_{s} \sin \left(\phi_{n, m}\right)+\Delta d_{u} \sin \left(\varphi_{n, m}\right)\right]}\right\} .
\end{aligned}
$$

The spatial CCFs observed at the MS for $\Delta d_{s}=0$ and the BS for $\Delta d_{u}=0$ can be expressed as

$$
\rho_{u_{1} u_{2}}^{M S}\left(\Delta d_{u}\right)=\frac{1}{M} \sum_{m=1}^{M} E\left\{e^{j k \Delta d_{u} \sin \left(\varphi_{n, m}\right)}\right\}
$$

and

$$
\rho_{s_{1} s_{2}}^{B S}\left(\Delta d_{s}\right)=\frac{1}{M} \sum_{m=1}^{M} E\left\{e^{j k \Delta d_{s} \sin \left(\phi_{n, m}\right)}\right\}
$$

respectively.

By imposing $\Delta d_{s}=0$ and $\Delta d_{u}=0$ in (10), we obtain the temporal ACF as

$$
r\left(\tau^{\prime}\right)=\frac{1}{M} \sum_{m=1}^{M} E\left\{e^{-j k\|\mathbf{v}\| \cos \left(\varphi_{n, m}-\theta_{v}\right) \tau^{\prime}}\right\} .
$$

2) Envelope LCR and AFD: The amplitude process $R(t)$ is obtained by taking the absolute value of the complex process $h_{u, s, n}(t)$, i.e., $R(t)=\left|h_{u, s, n}(t)\right|$. It can be shown that the envelope LCR can be expressed by [14]

$$
\begin{aligned}
N_{R}\left(r_{E}\right)= & 4 \pi p_{R}\left(r_{E}\right) \int_{0}^{\infty} \int_{0}^{\infty}\left[\prod_{m=1}^{M} J_{0}\left(4 \pi^{2}\left|f_{m} c_{m}\right| x\right)\right] \\
& \cdot J_{0}(2 \pi x y) x y^{2} d x d y
\end{aligned}
$$

where $c_{m}=\sqrt{\frac{P_{n}}{m}}$ and $f_{m}=v_{n, m}$ hold. Following the similar derivation procedure to that in [14]-[16], the amplitude PDF $p_{R}(z)$ can be expressed as

$$
p_{R}(z)=4 \pi^{2} z \int_{0}^{\infty}\left[\prod_{m=1}^{M} J_{0}\left(2 \pi c_{m} y\right)\right] J_{0}(2 \pi z y) y d y, z \geq 0 .
$$


If $M$ is sufficiently large, e.g., $M \geq 10$, the LCR in (15) can be approximated by [14]

$$
N_{R}\left(r_{E}\right) \approx \sqrt{\pi \sum_{m=1}^{M}\left(c_{m} f_{m}\right)^{2}} \cdot p_{R}\left(r_{E}\right) .
$$

The AFD is defined as $T_{R}\left(r_{E}\right)=\frac{P_{R}\left(r_{E}\right)}{N_{R}\left(r_{E}\right)}$ where $P_{R}\left(r_{E}\right)$ is the cumulative density function (CDF) of the amplitude process $R(t)$, i.e., $P_{R}\left(r_{E}\right)=\int_{0}^{r_{E}} p_{R}(z) d z$. Using (16), the $\mathrm{CDF} P_{R}\left(r_{E}\right)$ can be further expressed by $P_{R}\left(r_{E}\right)=4 \pi^{2} r_{E} \int_{0}^{\infty}\left[\prod_{m=1}^{M} J_{0}\left(2 \pi c_{m} y\right)\right] J_{1}\left(2 \pi r_{E} y\right) y d y$ [14], where $J_{1}(\cdot)$ is the first-order Bessel function of the first kind.

It makes sense to mention that, in case of the strongest clusters, the corresponding mathematical expressions for the statistical properties of the IMT-A channel model can be obtained by replacing the number of clusters $M$ by $M_{q}$ ( $\left.M_{1}=10, M_{2}=6, M_{3}=4\right)$ (see Table A1-6 on Page 39 in [12]), and/or the subscript $(n)$ by $(n, q)$.

\section{Comparison and Verification Results}

1) Spatial CCFs: The spatial CCFs of the IMT-A and LTEA channel models are investigated at three levels, namely, the cluster level, link level, and system level, as defined in [13]. Fig. 2 shows the absolute values of the cluster-level spatial CCFs at the MS and between two arbitrary channel coefficients of the LTE-A and IMT-A channel models. The mean AoA $\varphi_{n}$ (or $\widehat{\varphi}_{A o A}$ ) and mean $\mathrm{AoD} \phi_{n}$ (or $\widehat{\phi}_{A o D}$ ) are constant and equal $60^{\circ}$ for both channel models. Here, we considered the Urban Micro (UMi) scenario as defined in the IMT-A channel model, where MS cluster AoA spread (Cluster ASA) $=22^{\circ}$ and BS cluster AoD spread (Cluster ASD) $=10^{\circ}$ hold. It is clear that the spatial CCFs of IMT-A and LTE-A channel models at the cluster level do not match closely. Moreover, the spatial CCF of the IMT-A channel model fluctuates unstably around the LTE-A one. This is caused by the so-called "implementation loss" due to the insufficient number of subpaths used in the IMT-A channel model. A similar behavior for the SCM was previously highlighted in [13] where we suggested increasing the number of the subpaths in order to improve the simulation accuracy of the cluster-level spatial CCF at the MS.

In Fig. 3, using the UMi scenario with Cluster ASA= $22^{\circ}$ and Cluster $\mathrm{ASD}=10^{\circ}$, we show the absolute values of the link-level and system-level spatial CCFs at the MS and between two arbitrary channel coefficients versus the normalized MS antenna spacing $\Delta d_{u} / \lambda$ for both IMT-A and LTE-A channel models. For calculating link-level spatial CCFs, the line-of-sight $(\operatorname{LoS})$ AoA $\varphi_{L o S}$ and $\operatorname{LoS} \operatorname{AoD} \phi_{L o S}$ were kept constant and we used $\varphi_{L o S}=50^{\circ}$ and $\phi_{L o S}=195^{\circ}$, while the mean $\operatorname{AoA} \varphi_{n}$ (or $\widehat{\varphi}_{A o A}$ ) and mean $\operatorname{AoD} \phi_{n}$ (or $\widehat{\phi}_{A o A}$ ) are random variables. For calculating system-level spatial CCFs, both channel models used the same mean AoA/AoD generated randomly by the IMT-A channel model. The mean AoA $\varphi_{n}$ and mean AoD $\phi_{n}$ follow wrapped Gaussian distributions, while the $\operatorname{LoS} \operatorname{AoA} \varphi_{L o S}$ and $\operatorname{LoS} \operatorname{AoD} \phi_{L o S}$ follow uniform distributions. Fig. 3 clearly shows that both channel models

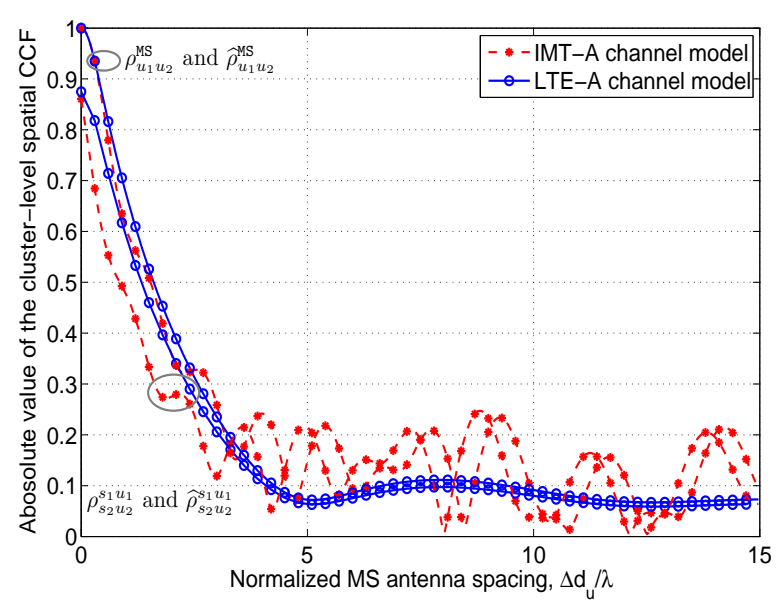

Fig. 2. The absolute values of the cluster-level spatial CCFs of the IMT-A and LTE-A channel models (UMi scenario, Cluster ASD = $10^{\circ}$, Cluster $\mathrm{ASA}=22^{\circ}, \Delta d_{s} / \lambda=1$, mean $\mathrm{AoD}=60^{\circ}$, mean $\mathrm{AoA}=$ $\left.60^{\circ}\right)$.

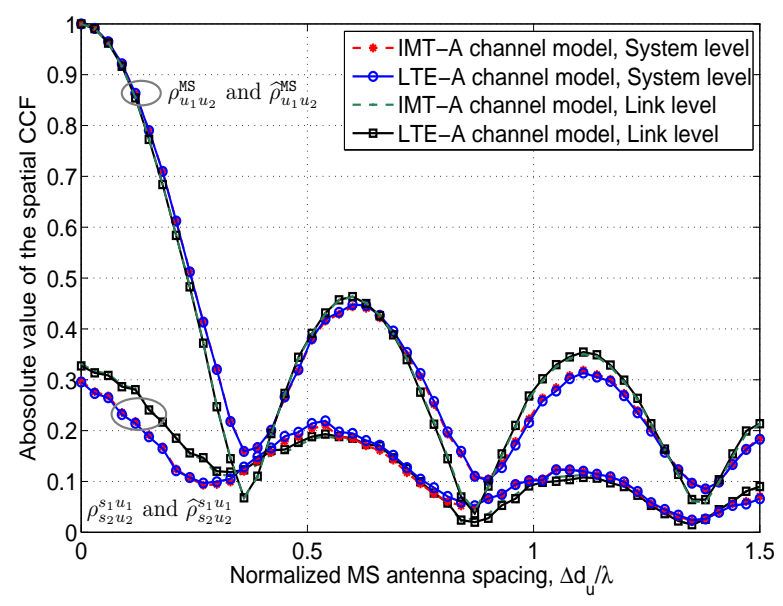

Fig. 3. The absolute values of the spatial CCFs of the IMT-A and LTE-A channel models at the link and system levels (UMi scenario, Cluster ASD $=10^{\circ}$, Cluster ASA $=22^{\circ}, \Delta d_{s} / \lambda=1, \phi_{L o S}=50^{\circ}$, $\left.\varphi_{L o S}=195^{\circ}\right)$.

tend to have very closely matched spatial CCFs at both the link and system levels, demonstrating their spatial separability or independence between the Tx and Rx.

2) Temporal ACFs: Fig. 4 shows the absolute values of the temporal ACFs of the LTE-A and IMT-A channel models, respectively, at three different levels. We still used the UMi scenario, while the MS speed was chosen as $1 \mathrm{~m} / \mathrm{s}$ and MS direction $\theta_{v}=60^{\circ}$. The ACF for the LTE-A channel model keeps the same at the three levels. Both models tend to have identical ACFs in the system level using the same parameters while the IMT-A channel model exhibits different behavior at cluster and link levels. This indicates that the spatial-temporal separability holds for the IMT-A channel model only at the system level, not at cluster and link levels, while it holds for the LTE-A channel model at the three levels. Similar to the conclusions in [13], the LTE-A channel model actually only 


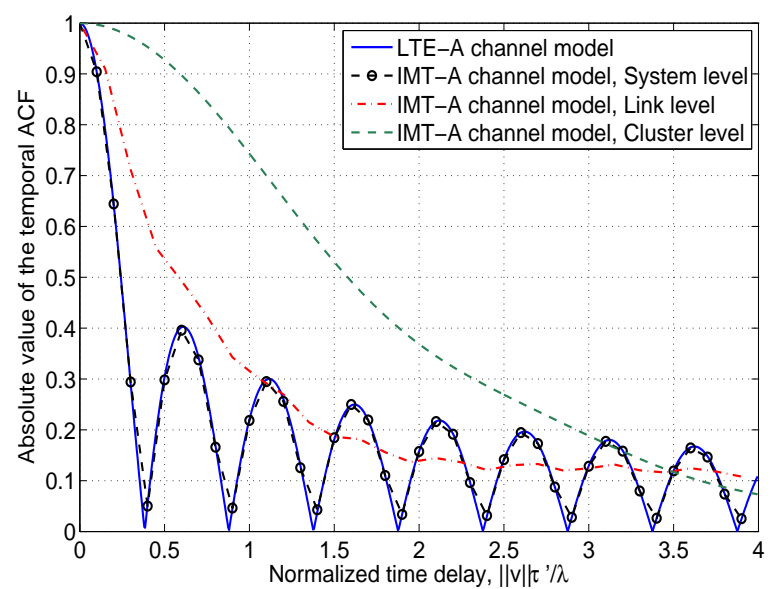

Fig. 4. The absolute values of the temporal ACFs of the LTE-A and IMT-A channel models at the cluster, link, and system levels $\left(\theta_{v}=60^{\circ}\right)$

models the average behavior of MIMO channels, while the IMT-A channel model provides us with more details regarding the variations across different realizations of MIMO channels.

It is important to mention that we have also obtained simulation results for the corresponding theoretical results in Figs. 2-4. They match very well, showing the correctness of our derivation and simulation results for spatial CCFs and temporal ACFs for both models. For clarity purposes, simulation results are not illustrated in Figs. 2-4.

3) Envelope LCRs and AFDs: Fig. 5 shows the theoretical normalized envelope LCRs for the LTE-A and IMT-A channel models, respectively, against the corresponding simulation results. The theoretical and simulated normalized AFDs for both models are shown in Fig. 6. Again, the simulation results closely match the corresponding theoretical results. This verifies the correctness of both theoretical derivations and simulations.

4) PDPs and FCFs: Fig. 7 illustrates the normalized FCFs of the LTE-A channel model for the Extended Pedestrian A (EPA) scenario and the IMT-A channel model for the UMi Non line-of-sight (NLoS) scenario. It is clear that the LTEA channel model only has acceptable performance below the $50 \mathrm{MHz}$ bandwidth, while cannot support bandwidths up to 100 MHz. The FCF of the IMT-A channel model has the period of $200 \mathrm{MHz}$ and is symmetrical with respect to $100 \mathrm{MHz}$.

\section{CONCLUSIONS}

We have thoroughly investigated the statistical properties of LTE-A and IMT-A channel models. Closed-form expressions have been derived for the spatial CCFs, temporal ACFs, envelope LCRs, AFDs, PDPs, and FCFs, verified by the corresponding simulation results. In general, the LTE-A channel model has much less model parameters and therefore is simpler than the IMT-A channel model. However, the LTEA channel model has significant flaws in terms of the model accuracy. From its PDP and FCF, it is clear that the LTEA channel model can only support system bandwidths up

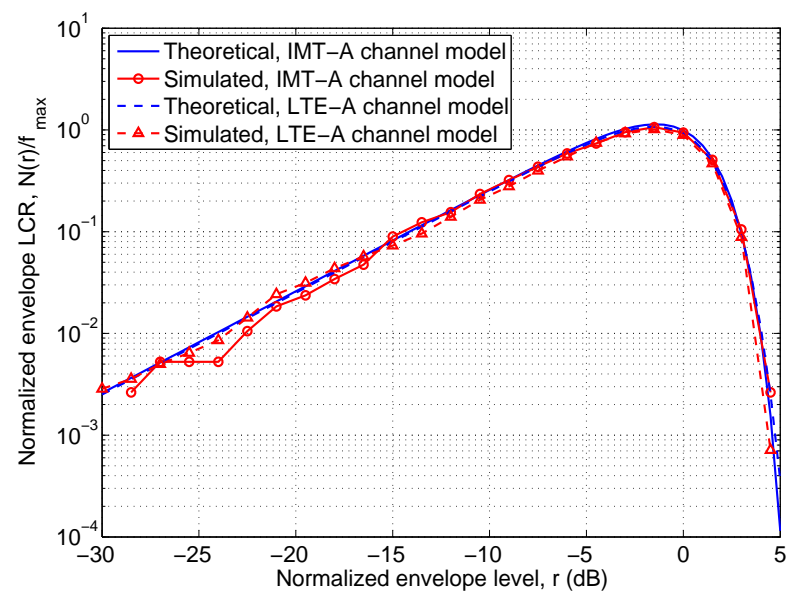

Fig. 5. The normalized envelope LCRs for the LTE-A and IMT-A channel models $\left(P_{n}=2 \sigma^{2}=1, M=20\right)$.

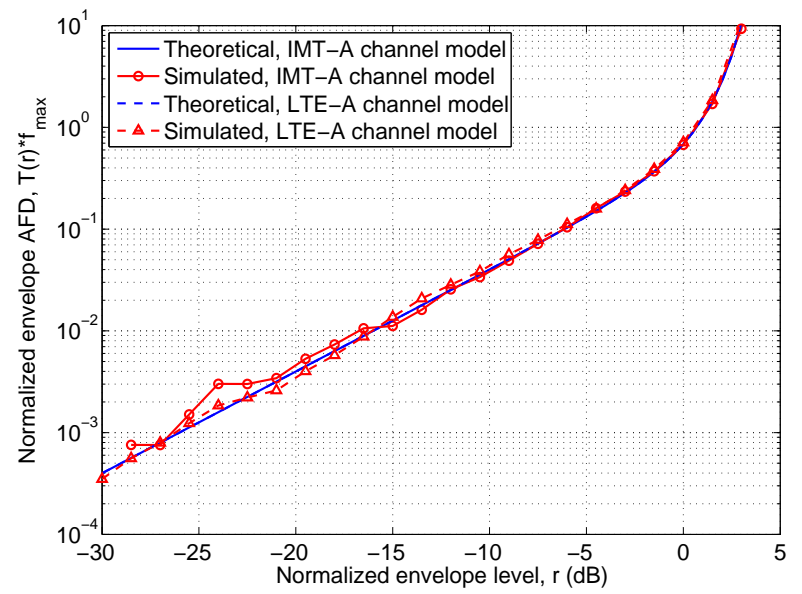

Fig. 6. The normalized envelope AFDs for the LTE-A and IMT-A channel models $\left(P_{n}=2 \sigma^{2}=1, M=20\right)$.

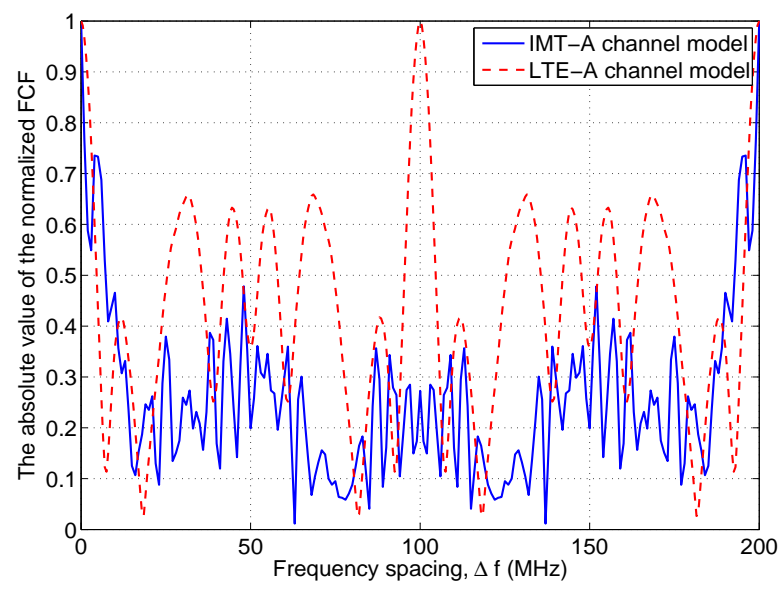

Fig. 7. The normalized FCFs of the LTE-A channel model (EPA NLoS scenario) and the IMT-A channel model (UMi NLoS scenario).

to $50 \mathrm{MHz}$, not the claimed $100 \mathrm{MHz}$. Also, the LTE-A 
channel model has the spatial separability and spatial-temporal separability at all the three levels, describing only the average spatial-temporal properties of MIMO channels. For the IMTA channel model, the spatial separability can be observed only at the link and system levels, while the spatial-temporal separability can be observed only at the system level. This means that the IMT-A channel model is more statistically accurate as it allows us to simulate the variations of different MIMO channel realizations. From its PDP and FCF, the IMTA channel model can indeed support system bandwidths up to $100 \mathrm{MHz}$.

\section{ACKNOWLEDGMENTS}

This work is sponsored by Huawei Technologies. The authors would like to acknowledge the support from the RCUK for the UK-China Science Bridges Project: R\&D on (B)4G Wireless Mobile Communications. Q. Yao, Y. Yuan, A. Ghazal, and C.-X. Wang would also like to acknowledge the support from the Scottish Funding Council for the Joint Research Institute in Signal and Image Processing with the University of Edinburgh, as part of the Edinburgh Research Partnership in Engineering and Mathematics (ERPem).

\section{REFERENCES}

[1] I. E. Talatar, "Capacity of multi-antenna Gaussian channels," Tech. Rep., AT \& T Bell Labs, Florham Park, NJ, USA, Jun. 1995.

[2] G. J. Foschini and M. J. Gans, "On limits of wireless communications in a fading environment when using multiple antennas," Wireless Personal Commun., vol. 6, no. 3, pp. 311-335, Mar. 1998.

[3] J. P. Kermoal, L. Schumacher, K. I. Pedersen, P. E. Mogensen and F. Frederiksen, "A stochastic MIMO radio channel model with experimental validation," IEEE J. Selected Areas in Commun., vol. 20, no. 6, pp. 12111226, Aug. 2002.

[4] V. Erceg, et al., IEEE 802.11 document 03/940r4, "TGn Channel Models,", May 2004.

[5] Motorola, etc., 3GPP, R4-071444, "Text proposal to 36.803 for MIMO correlation matrices," Aug. 2007.

[6] Agilent etc., 3GPP, R-071825, "Proposal for MIMO correlation matrices," Nov. 2007.

[7] 3GPP, TS36.101, V8.13.1, "3rd Generation Partnership Project; Technical Specification Group Radio Access Network; Evolved Universal Terrestrial Radio Access (E-UTRA); User Equipment (UE) radio transmission and reception (Release 8)," Apr. 2011.

[8] 3GPP, TS36.101, V10.2.1, "3rd Generation Partnership Project; Technical Specification Group Radio Access Network; Evolved Universal Terrestrial Radio Access (E-UTRA); User Equipment (UE) radio transmission and reception (Release 10)," Apr. 2011.

[9] 3GPP TR 25.996 v10.0.0, "spatial channel model for multiple input multiple output (MIMO) simulations," Release 10, Mar. 2011.

[10] P. Kyösti, et al., "WINNER II channel models," IST-4-027756, WINNER II D1.1.2, v1.2, Apr. 2008

[11] ITU-R M.2135, "Guidelines for Evaluation of Radio Interface Technologies for IMT-Advanced," Geneva, Switzerland, Rep. ITU-R M.2135, 2008.

[12] ITU-R M.2135-1, "Guidelines for Evaluation of Radio Interface Technologies for IMT-Advanced," Geneva, Switzerland, Rep. ITU-R M.21351, Dec. 2009

[13] C.-X. Wang, X. Hong, H. Wu, and W. Xu, "Spatial temporal correlation properties of the 3GPP spatial channel model and the Kronecker MIMO channel model," EURASIP J. Wireless Commun. and Networking, vol. 2007, Article ID 39871, 9 pages, 2007. doi:10.1155/2007/39871.

[14] M. Pätzold and C. A. Gutiérrez, "Level-crossing rate and average duration of fades of the envelope of a sum-of-cisoids," in Proc. VTC'08Spring, Singapore, May 2008, pp. 488-494.
[15] C.-X. Wang, N. Youssef, and M. Pätzold, "Level-crossing rate and average duration of fades of deterministic simulation models for NakagamiHoyt fading channels," In Proc. WPMC'02, Honolulu, Hawaii, Oct. 2002, pp. 272-276.

[16] M. Pätzold and B. Talh, "On the statistical properties of sum-of-cisoidsbased mobile radio channel models," in Proc. WPMC'07, Jaipur, India, Dec. 2007, pp. 394-400. 